\title{
Plaquette Valence Bond Theory of Cuprate High-Temperature Superconductivity
}

\author{
Malte Harland $^{1, *}$, Tim Berberich ${ }^{1}$, Mikhail I. Katsnelson ${ }^{2}$, Alexander I. Lichtenstein ${ }^{1,3}$ \\ ${ }^{1}$ Institute of Theoretical Physics, University of Hamburg, Hamburg, 20355, Germany \\ ${ }^{2}$ Institute for Molecules and Materials, Radboud University, Nijmegen, 6525AJ, the Netherlands \\ ${ }^{3}$ The Hamburg Centre for Ultrafast Imaging, Hamburg, 22761, Germany
}

Copyright $\subseteq 2018$ by authors, all rights reserved. Authors agree that this article remains permanently open access under the terms of the Creative Commons Attribution License 4.0 International License

\begin{abstract}
We investigate the phenomenon of high-temperature superconductivity within a strong coupling perspective. The occurence is traced to a quantum critical point that is in the phase diagram of the plaquette's $t, t^{\prime}$-Hubbard model. We develop a bottom-up approach combining several methods, i.e. exact diagonalization of an isolated plaquette, the Lanczos-method for a plaquette within a bath and cluster dynamical Mean-Field theory with continuous time quantum Monte-Carlo solver to embedd the plaquette in a lattice environment. The quantum critical point is located where the $N=2,3,4$-sectors of the plaquette cross. This point is also found to show

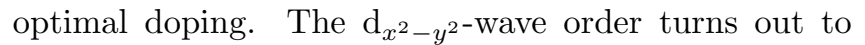
be largest at the localized-itinerant transition of the electrons. Furthermore, we present an explenation for the pseudo-gap phenomenon, that is explained by a soft mode related to local singlets of the plaquette. The theory presented here is similar to the resonating valence bond theory, but stresses the importance of local singlets.
\end{abstract}

Keywords Quantum Critical Point, Plaquette, Hubbard Model, Resonating Valence Bonds

\section{Introduction}

After 30 years history of extremely intensive experimental [1-4] and theoretical [5-10] studies of the hightemperature superconductivity (HTSC) in copper oxides we are still far from understanding the basic mechanism of this fascinating phenomenon. Taking into account the enormous number of researchers involved in this field, one can assume that almost all possible ideas were expressed and that the main problem is just to select the basic simple concepts from the pile of available theoretical results. The most ambitious attempt was made by $\mathrm{P}$. W. Anderson who emphasized with his resonating valence bond (RVB) theory the crucial importance of strong electron correlations, the tendency to singlet spin state formation and the non-Fermi liquid character of the normal phase [6]. Unfortunately, details of his original approach, such as suppression of interlayer hopping in the normal phase as the main factor of superconductivity, seems to contradict experimental data [11]. The latest version of the RVB theory is presented in [12]. We believe, that the main assumption of the strongly correlated limit as the base of understanding the hightemperature superconductivity is correct, as well as emphasizing a crucial role of spin singlet states, but important details were missing. Below we present arguments for the thesis, that the minimal object of HTSC-theory is the plaquette in the so-called effective $t, t^{\prime}$ Hubbard model [13], rather than the conventional atomic limit typical for the theory of Mott insulators $[6,9]$. The best practical realization of this atomic based theory is the dynamical mean-field theory (DMFT) [14]. The obvious minimal generalization in the case of $\mathrm{d}_{x^{2}-y^{2} \text {-wave }}$ pairing is a cluster DMFT (CDMFT) scheme $[15,16]$.

Since the first plaquette CDMFT calculation of $\mathrm{d}_{x^{2}-y^{2}}$-wave superconducting order together with antiferromagnetic fluctuations [15], there have been many calculations for different cluster sizes and geometries based on continuous-time Quantum Monte Carlo (CTQMC) or exact diagonalization (ED) solvers [17$23,16,24-31$. Unfortunately, the basic qualitative feature of the many-body states in the plaquette were hidden in computational details. The main aim of this work is to present a simple and transparent strong coupling

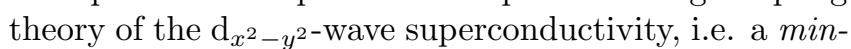
imal consistent many-body model, based on the plaquette energy spectrum peculiarity, namely the "quantum critical point", that merges two singlets and two doublets. These states of the doped plaquette are different from those discussed in the resonating valence-bond theory $[6,12]$. The main point is that the quantum critical point discussed here is related to the formation of local valence bonds in the frustrated quantum spin model [32]. Therefore, the optimal superconducting states are located on the border between localized and delocalized resonating plaquette valence bonds. Here we follow a bottom-up approach starting with an isolated plaquette 
and building stepwise a more complex environment.

An important theoretical problem is to find a minimal and generic electronic-structure model of cuprate superconductors. From band structure calculations [13] we can safely reduce it to an effective one-band model with long-range hopping. We use a standard parametrization of the tight-binding model for $\mathrm{YBa}_{2} \mathrm{Cu}_{3} \mathrm{O}_{7}[13]$ with the next-nearest neighbor hopping: $t^{\prime} / t=-0.3$ and $t$ as unit of our energies. The local Hubbard interaction parameter $U$ is of the order of the bandwidth $W=8 t$. Then, the $t, t^{\prime}$ Hubbard model on the square lattice reads

$$
H=-\sum_{i j} t_{i j} c_{i \sigma}^{\dagger} c_{j \sigma}+\sum_{i} U n_{i \uparrow} n_{i \downarrow}
$$

where $t_{i j}$ is an effective hopping and $U$ the local Coulomb interaction. The operators $c_{i \sigma}^{\dagger}, c_{i \sigma}$ create and annihilate fermions at site $i$ with $\operatorname{spin} \sigma=\uparrow(+), \downarrow(-)$, respectively and the occupation operator is $n_{i \sigma}=c_{i \sigma}^{\dagger} c_{i \sigma}$.

\section{Isolated Plaquette}

We start the discussion with electronic states in the isolated Hubbard plaquette. The optimal doping for high-temperature superconductivity is of the order of $15 \%$ of holes per site for almost all cuprate materials. This gives us an average number of electrons per site of 0.85 and results in 3.4 fermions per 4 -site plaquette in the crystal. We argue, that this is related to 3-electron states of the isolated plaquette, since particle-hole asymmetry introduced by the next-nearest neighbor hopping $t^{\prime}$, with moderate values of $U$ and certain fixed chemical potentials $(\mu)$ result in an occupation per plaquette of the crystal, that is very close to the optimal value of 3.4 electrons.

The Hamiltonian of the isolated plaquette reads

$$
\begin{gathered}
H_{p}=\sum_{(i, j)=1 . .4} h_{i j}^{0} c_{i \sigma}^{\dagger} c_{j \sigma}+\sum_{i=1 . .4} U n_{i \uparrow} n_{i \downarrow}, \\
-\hat{h}_{0}=\left(\begin{array}{cccc}
\mu & t & t^{\prime} & t \\
t & \mu & t & t^{\prime} \\
t^{\prime} & t & \mu & t \\
t & t^{\prime} & t & \mu
\end{array}\right)
\end{gathered}
$$

We include the chemical potential in the diagonal part of $h_{i j}^{0}$. We present in Fig. 1 regions in the $U-\mu$ space, whose ground states have an occupation of three plusminus one electrons. The one-electron spectrum possesses four states with the energies: $\pm 2 t-t^{\prime}-\mu$ and double-degenerate $t^{\prime}-\mu$. At zero interaction $U=0$, there is no stable ground state with three electrons, in the sense that one can add or remove one electron without changing the thermodynamic potential. Starting from some critical interaction strength $U \approx 3$ there is a small region, in that the plaquette ground state with $N=3$ electrons is separated by energy gaps from the states with $N=2$ and $N=4$, see Fig. 1 . Importantly, this $N=3$ ground state is fourfold degenerate consisting of two doublets in the sectors $\left(2_{\uparrow}, 1_{\downarrow}\right)$ and $\left(1_{\uparrow}, 2_{\downarrow}\right)$, which we label $|X\rangle$ and $|Y\rangle$, according to their symmetry. Moreover, there is a critical point (circle in Fig. 1) where all three sectors with 2,3 , and 4 electrons have the same ground state energy and form a sixfold degenerate ground-state multiplet consisting of two singlets of the sectors $\left(1_{\uparrow}, 1_{\downarrow}\right)$ and $\left(2_{\uparrow}, 2_{\downarrow}\right)$ together with two doublets of the 3 -electron sectors. This critical point has the coordinates $U=2.78, \mu=0.24$ for the standard value of $t^{\prime} / t=-0.3$. At smaller $t^{\prime}$ this critical point shifts to larger $U$ and at $t^{\prime} / t=0$ its coordinates are $U=4.58, \mu=0.72$. We think, that this critical point of the plaquette has crucial importance for the physics of the strong-coupling $\mathrm{d}_{x^{2}-y^{2}}$-wave superconductivity. Here, we demonstrate via bottom-up approach, that this is the key ingredient of a consistent minimal picture of HTSC.

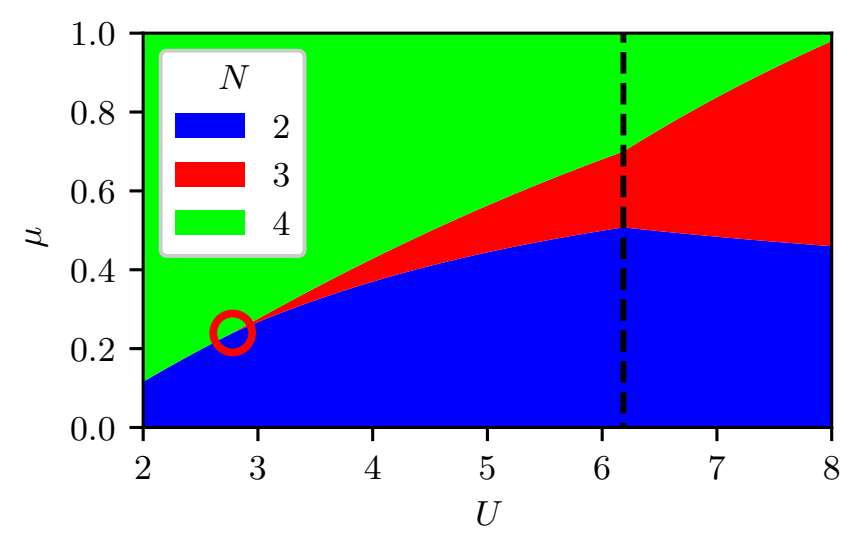

Figure 1. Zero-temperature phase diagram of the isolated plaquette as function of Hubbard $U$ and chemical potential $\mu$ in proximity of the quantum critical point (circle) for $t^{\prime} / t=-0.3$.

If we approach this critical point from the region with the $N=3$ ground state, then the one electron density of states (DOS) at the Fermi energy diverges for both, electron and hole sides, due to transitions from the fourfold degenerate $N=3$ ground state to singlets of $N=2$ (hole side) and $N=4$ (electron side) with zero excitation energy. The corresponding spectral weights with normalization of the $\delta$-functions are equal to 0.46 and 0.23 for hole and electron sides, respectively. Thus, it introduces an important electron-hole asymmetry.

In Fig. 2 we show static observables of the plaquette in very close proximity to the critical point. The local charge is denoted by circles and the nearest-neighbor Heisenberg-spin correlations by strips. Shown are all six states of the different particle number sectors. Dark (light) circles denote electrons (holes) occupying the respective site and dark (light) strips triplet (singlet)like correlations. Regarding this set of observables, the $N=2$ and the $N=4$ states obey the $Z_{4}$-symmetry. But the spin-doublets of $N=3$ reduce it to $Z_{2}$, which is exactly the $d$-wave symmetry. Due to the rotation symmetry, we can relate the orbitals of the plaquette to high-symmetry points of the lattice's Brillouin zone, i.e. $G, X, Y$ and $M$. Although $d$-wave pairing takes place in the antinodal $X$ and $Y$ directions, we learn from the doublets in Fig. 2, that correlations of the nodal direction $M$ are involved, which agrees with recent experimental results. $[33,34]$ The $Z_{2}$ symmetry of the $N=3$ states changes to $Z_{4}$ at $U=6.18$ (dashed line in Fig. 1), where also the slope of the phase boundaries changes discontinuously indicating a groundstate crossover within the $N=3$ sector. The fourfold degeneracy is preserved for $U>6.18$ and the nearest neighbor spin correlations become triplet-like, signaling a localization process with supression of charge fluctuations, that 
is understood for large $U$ by e.g. mapping to the $t-J$ model[17]. A calculation of a plaquette observable with exact-diagonalization does always weighten the degenerate $N=3$ states equally and their superposition preseves the plaquette's symmetry. However, a coupling to another system has potetntial for creating modes that resonate stronger with one of the doublet states than with the others.
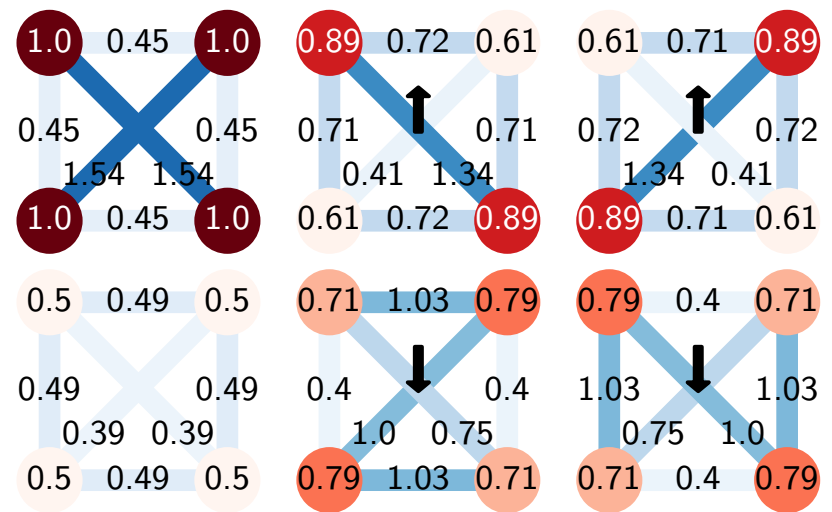

Figure 2. Static observables of the local charge (circles) and the nearest-neighbor Heisenberg-spin correlations(strips) in proximity of the critical point for $N=2$ (top left), $N=4$ (bottom left) and the four-fold degenerate $N=3$ (mid, right). In case of the doublets the total spin along quantization axis is indicated by a black arrow.

\section{Plaquette in Bath}

The appearance of the DOS peak at the Fermi energy leads to a universal instability in the sense, that the susceptibility diverges in many different channels (magnetic, superconducting, charge density wave, etc.). In order to study the interplay of different instability channels from the strong-coupling perspective we introduce a simple model of an embedded plaquette in the spirit of DMET [35]. To this aim we add to the plaquette's Hamiltonian a hybridization with four fermionic bath states, one bath state per corner of the plaquette. We use an exact diagonalization technique, namely the Lanczos scheme with a Hilbert space size of $2^{16}$, without any symmetry restrictions. Furthermore, we introduce different symmetry breaking fields acting on the bath

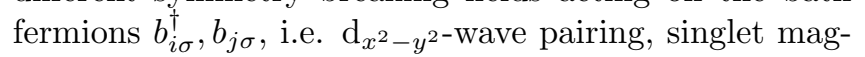
netic states on the bonds, and the conventional Néel antiferromagnetic state:

$$
\begin{aligned}
h_{d} & =\sum_{\sigma=\uparrow, \downarrow, i=1 . .4}(-1)^{i} \sigma \Delta_{d}\left(b_{i, \sigma} b_{i+1,-\sigma}+\text { h.c. }\right), \\
h_{s} & =\sum_{\sigma=\uparrow, \downarrow, i=1 . .4}(-1)^{i} \sigma \Delta_{s}\left(b_{i, \sigma}^{\dagger} b_{i+1,-\sigma}+\text { h.c. }\right), \\
h_{m} & =\frac{1}{2} \sum_{\sigma=\uparrow, \downarrow, i=1 . .4}(-1)^{i} \sigma \Delta_{m} b_{i, \sigma}^{\dagger} b_{i, \sigma} .
\end{aligned}
$$

Here we assume periodic boundary conditions, that means for $i=4$ we define $i+1=1$. We switch on small fields $\Delta_{d}=\Delta_{s}=\Delta_{m}=0.01 t$ simultaneously and calculate numerically their associated susceptibilities of the plaquette. The hybridization $V$ between the fermions $c_{i \sigma}^{\dagger}$ and $b_{j \sigma}^{\dagger}$ breaks the sixfold degeneracy of the plaquette's quantum critical point and without external fields it results in a singlet ground state. The $\mathrm{d}_{x^{2}-y^{2-}}$ wave superconducting 3 and the magnetic bond-singlet 4 external fields respect quantum entanglement of the singlet character of the ground state, whereas the Néel field 5 destroys it. Being classical in its nature, the Néel state is expected to be most stable for sufficiently strong coupling with the environment $V[36]$ or high temperatures [37].

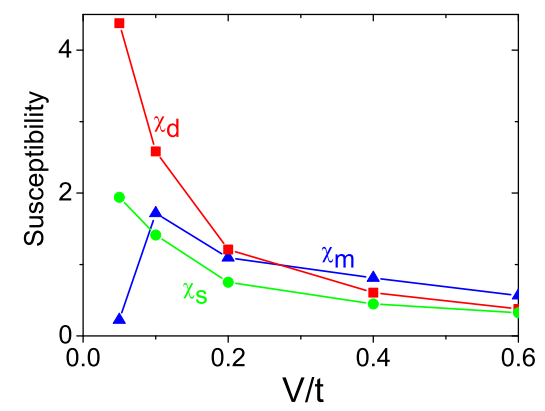

Figure 3. Superconducting $\left(\chi_{d}\right)$, singlet bond order $\left(\chi_{s}\right)$ and antiferromagnetic $\left(\chi_{m}\right)$ susceptibility of the plaquette in a bath as a function of the hybridization $V$ for $U=3$ and $\mu=0.27$.

For an infinite system different types of order can be found by studying divergences of susceptibilities. Since in DMET we deal with finite systems, the susceptibilities remain finite up to zero temperature and we assume, that the largest susceptibility of the cluster signals the corresponding order of the crystal. The computational results are shown in Fig. 3 as function of the hybridization parameter $V$. One can see, that $\mathrm{d}_{x^{2}-y^{2} \text {-wave su- }}$ perconducting pairing always wins in comparison with the singlet bond pairing and is more favorable than the Néel order for $V \leq 0.2$. The self-consistent plaquetteBethe DMFT for the cluster case (see below) with optimal HTSC parameters corresponds to $V=0.1$. The singlet ground state near the plaquette's quantum critical point favors $\mathrm{d}_{x^{2}-y^{2}}$-wave superconductivity, rather than magnetic ordering. This result agrees well with large scale CDMFT calculations for optimal doping [27, 28].

There are three main contributions by the singleplaquette sectors with $N=2,3,4$ electrons, which have almost equal spectral weights. In particular, it means a strong mixture of states, that differ by two electrons, i.e. by a local Cooper pair on the bond. This is understood as an important detail for superconductivity, since the degenerate quantum critical point (circle in Fig. 1) consists of states with the same total number of electrons $\left(N_{t o t}=8\right)$, but none of those shows a double occupation on the plaquette. It occurs only on bath sites, that have no Hubbard interaction. The degeneracy of the quantum critical point splits into four states for $N=2$, eight states for $N=3$ and two states for $N=4$. Taking into account the total number of these states and including all their antisymmetric singlet combinations, we observe, that $85 \%$ of the ground state components are related to the quantum critical point. This means, that the system as a whole perfectly screens the strong Coulomb interactions (Hubbard $\mathrm{U}$ is about $70 \%$ of the bandwidth) and the plaquette states with $N=2,3,4$ electrons contribute equally to the ground state singlet of the total system. A specific symmetry of the ground state at the 
plaquette's quantum critical point increases effectively the single-site $U$ by suppressing double occupations on plaquette sites. But, at the same time, it decreases the effective $U$ for the plaquette in a sense, that one does not have to pay an additional energy for adding two more electrons to the plaquette as a whole. This situation is very nontrivial; it demonstrates clearly, that discussions of strong correlations for the high-Tc cuprates would be based on a rather four-orbital Hubbard model, corresponding to a lattice built from plaquettes, than on an initial single-band Hubbard model for copper sites.

\section{Plaquette CDMFT}

Finally, we perform the standard CDMFT calculations using a strong-coupling continuous-time Quantum Monte Carlo solver [38, 39] in the normal state. Since a transition to the periodic plaquette in the crystal increases the bandwidth by a factor of two due to doubling of the coordination numbers compared to the isolated plaquette, we increase the values of $U$ and $\mu$ by the factor of two. Note, that the natural energy unit is the bandwidth $W$, rather than the hopping $t$. Furthermore, we use the value of $U / t=6$, which approximately describes real cuprate materials [40].

The calculated local DOS obtained by maximumentropy analytic continuation [41] is shown in Fig. (4). We observe, that for sufficiently high temperatures there is a broad peak at the Fermi energy originating from the plaquette quantum critical point. This relation is illustrated in the inset of Fig. (4). We artificially scaled the hopping between plaquettes by a factor $\alpha$ ranging from 0 to the physical value 1 .

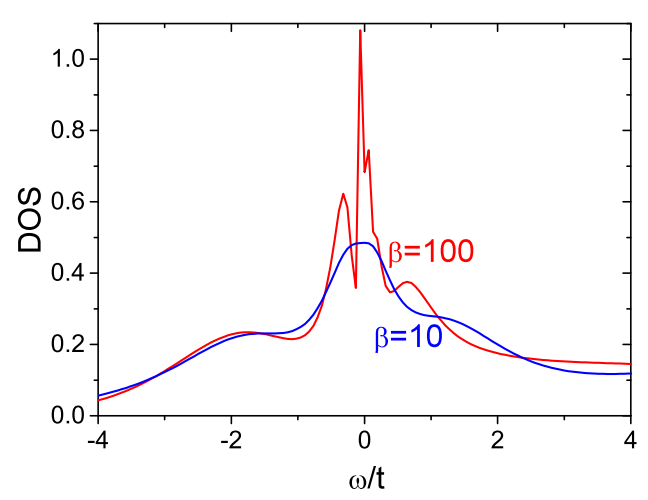

Figure 4. Density of states for both spins of the plaquette CDMFT for $U=6$ and $\mu=0.54$ for different temperatures.

At lower temperatures a pseudogap is formed. It is well-known in all HTSC materials and considered as one of its most striking features [42]. Sometimes this pseudogap is also considered to be the precursor of the superconducting gap (formation of incoherent Cooper pairs above $T_{c}$ ) or as a smeared antiferromagnetic gap (shadow bands) [42]. However, both interpretations have problems, when they get compared to experiments [42]. Within our scheme it is natural to interpret this pseudogap as a pseudo-hybridization-gap similar to that arising in Kondo lattices [43] or intermediate valence semiconductors [44]. From this point of view the pseudogap in HTSC materials originates from the Fano antires- onance due to embedding of the soft-fermion mode of the plaquette (discussed above) into a continuous band spectrum of the lattice. The role of soft-fermion modes in a different context, the hidden fermion, has been discussed in $[45]$.

The density of states for optimal doping $n=0.85$ in the self-consistent CDMFT calculations is shown in the right inset of Fig. 4. Its chemical potential converges to a value of $\mu=1.2$, which is very similar to that of the fixed $\mu$ of the quantum critical point in the isolated plaquette. Furthermore, we have calculated a low-temperature superconducting state with CDMFT, Lanczos solver and 8-bath sites and also with a CTQMC cluster solver and found similar results to many other calculations $[17,19,20,23,16,24-29]$.

\section{Conclusions}

To conclude, we developed a picture of HTSC based on the existence of a quantum critical point at the crossing of the ground state energies in the $N=2,3,4$ sectors within the plaquette for parameters close to the optimal doping, $t^{\prime}$ being of crucial importance. Contrary to the original resonating valence bond (RVB) theory of Anderson [6], we start with the local valence bond formation in the doped plaquette. The optimal superconducting region is related to a localized-delocalized transition of plaquette valence bond states in the plaquette. It would be interesting to describe the formation of global singlet states with plaquette valence bond states using the matrix product scheme [46], since the CDMFT scheme breaks translational symmetry.

Formation of the soft-fermion mode near the optimal doping has an analog in the weak coupling theory within the van Hove scenario of HTSC [47, 48]. Due to the formation of flat bands of many-body origin [47] there is a whole region of parameters $t^{\prime}, U, \mu$, in that the Fermiliquid description is broken. However, we believe that the strong-coupling description presented here is more relevant for real HTSC materials, which are characterized by large values of $U$ [40].

\section{Acknowledgements}

A. I. L. acknowledges support from the DFG SFB668 and The Hamburg Centre for Ultrafast Imaging, M. I. K. acknowledges financial support from ERC (project 338957 FEMTO/NANO) and from NWO via the Spinoza Prize. Computations have been performed at the NIC, Forschungszentrum Jülich, under project HHH26.

\section{REFERENCES}

[1] J. G. Bednorz and K. A. Müller. Possible hightc superconductivity in the balacuo system. Zeitschrift für Physik B Condensed Matter, 64(2):189-193, 1986.

[2] Andrea Damascelli, Zahid Hussain, and Zhi-Xun Shen. Angle-resolved photoemission studies of the 
cuprate superconductors. Rev. Mod. Phys., 75:473541, Apr 2003.

[3] J. Orenstein and A. J. Millis. Advances in the physics of high-temperature superconductivity. Science, 288(5465):468-474, 2000.

[4] M. H. Hamidian, S. D. Edkins, Chung Koo Kim, J. C. Davis, A. P. Mackenzie, H. Eisaki, S. Uchida, M. J. Lawler, E.-A. Kim, S. Sachdev, and K. Fujita. Atomic-scale electronic structure of the cuprate dsymmetry form factor density wave state. Nat Phys, 12(2):150-156, February 2016.

[5] P.W. Anderson. A re-examination of concepts in magnetic metals: The nearly antiferromagnetic fermi liquid. Advances in Physics, 46(1):3-11, 1997.

[6] Philip W Anderson et al. The theory of superconductivity in the high-Tc

cuprate superconductors, volume 446. Princeton University Press Princeton, NJ, 1997.

[7] D. J. Scalapino. A common thread: The pairing interaction for unconventional superconductors. Rev. Mod. Phys., 84:1383-1417, Oct 2012.

[8] Jrg Schmalian, David Pines, and Branko Stojkovi. Weak pseudogap behavior in the underdoped cuprate superconductors. Journal of Physics and Chemistry of Solids, 59(1012):1764 - 1768, 1998.

[9] Masatoshi Imada, Atsushi Fujimori, and Yoshinori Tokura. Metal-insulator transitions. Rev. Mod. Phys., 70:1039-1263, Oct 1998.

[10] Elbio Dagotto. Correlated electrons in hightemperature superconductors. Rev. Mod. Phys., 66: 763-840, Jul 1994.

[11] H. J. A. Molegraaf, C. Presura, D. van der Marel, P. H. Kes, and M. Li. Superconductivityinduced transfer of in-plane spectral weight in bi2sr2cacu2o8+. Science, 295(5563):2239-2241, 2002.

[12] PHILIP W. ANDERSON. Personal history of my engagement with cuprate superconductivity, 19862010. International Journal of Modern Physics B, 25(01):1-39, 2011.

[13] O.K. Andersen, A.I. Liechtenstein, O. Jepsen, and F. Paulsen. Proceedings of the conference on spectroscopies in novel superconductors lda energy bands, low-energy hamiltonians, t, t, t (k), and j. Journal of Physics and Chemistry of Solids, 56(12): 1573 - 1591, 1995.

[14] Antoine Georges, Gabriel Kotliar, Werner Krauth, and Marcelo J. Rozenberg. Dynamical mean-field theory of strongly correlated fermion systems and the limit of infinite dimensions. Rev. Mod. Phys., 68:13-125, Jan 1996.

[15] A. I. Lichtenstein and M. I. Katsnelson. Antiferromagnetism and $\boldsymbol{d}$-wave superconductivity in cuprates: A cluster dynamical mean-field theory. Phys. Rev. B, 62:R9283-R9286, Oct 2000.
[16] Thomas Maier, Mark Jarrell, Thomas Pruschke, and Matthias H. Hettler. Quantum cluster theories. Rev. Mod. Phys., 77:1027-1080, Oct 2005.

[17] Kristjan Haule and Gabriel Kotliar. Strongly correlated superconductivity: A plaquette dynamical mean-field theory study. Phys. Rev. B, 76:104509, Sep 2007.

[18] M. Ferrero, P. S. Cornaglia, L. De Leo, O. Parcollet, G. Kotliar, and A. Georges. Valence bond dynamical mean-field theory of doped mott insulators with nodal/antinodal differentiation. EPL (Europhysics Letters), 85(5):57009, 2009.

[19] Michel Ferrero, Pablo S. Cornaglia, Lorenzo De Leo, Olivier Parcollet, Gabriel Kotliar, and Antoine Georges. Pseudogap opening and formation of fermi arcs as an orbital-selective mott transition in momentum space. Phys. Rev. B, 80:064501, Aug 2009 .

[20] Emanuel Gull, Olivier Parcollet, and Andrew J. Millis. Superconductivity and the pseudogap in the two-dimensional hubbard model. Phys. Rev. Lett., 110:216405, May 2013.

[21] E. Khatami, K. Mikelsons, D. Galanakis, A. Macridin, J. Moreno, R. T. Scalettar, and M. Jarrell. Quantum criticality due to incipient phase separation in the two-dimensional hubbard model. Phys. Rev. B, 81:201101, May 2010.

[22] E. Gull, M. Ferrero, O. Parcollet, A. Georges, and A. J. Millis. Momentum-space anisotropy and pseudogaps: A comparative cluster dynamical meanfield analysis of the doping-driven metal-insulator transition in the two-dimensional hubbard model. Phys. Rev. B, 82:155101, Oct 2010.

[23] David Sénéchal, P.-L. Lavertu, M.-A. Marois, and A.-M. S. Tremblay. Competition between antiferromagnetism and superconductivity in high- $T_{c}$ cuprates. Phys. Rev. Lett., 94:156404, Apr 2005.

[24] T. A. Maier, M. S. Jarrell, and D. J. Scalapino. Structure of the pairing interaction in the twodimensional hubbard model. Phys. Rev. Lett., 96: 047005 , Feb 2006.

[25] M. Aichhorn, E. Arrigoni, M. Potthoff, and W. Hanke. Phase separation and competition of superconductivity and magnetism in the twodimensional hubbard model: From strong to weak coupling. Phys. Rev. B, 76:224509, Dec 2007.

[26] E. Gull, P. Werner, X. Wang, M. Troyer, and A. J. Millis. Local order and the gapped phase of the hubbard model: A plaquette dynamical mean-field investigation. EPL (Europhysics Letters), 84(3): $37009,2008$.

[27] M. Civelli. Evolution of the dynamical pairing across the phase diagram of a strongly correlated high-temperature superconductor. Phys. Rev. Lett., 103:136402, Sep 2009. 
[28] S. S. Kancharla, B. Kyung, D. Sénéchal, M. Civelli, M. Capone, G. Kotliar, and A.-M. S. Tremblay. Anomalous superconductivity and its competition with antiferromagnetism in doped mott insulators. Phys. Rev. B, 77:184516, May 2008.

[29] Satoshi Okamoto and Thomas A. Maier. Enhanced superconductivity in superlattices of high$T_{c}$ cuprates. Phys. Rev. Lett., 101:156401, Oct 2008.

[30] Xi Chen, J. P. F. LeBlanc, and Emanuel Gull. Superconducting fluctuations in the normal state of the two-dimensional hubbard model. Phys. Rev. Lett., 115:116402, Sep 2015.

[31] L Fratino, P Smon, G Sordi, and A-M S Tremblay. An organizing principle for two-dimensional strongly correlated superconductivity. Scientific Reports, 6:22715-, February 2016.

[32] I Affleck. Exact results on the dimerisation transition in $\mathrm{su}(\mathrm{n})$ antiferromagnetic chains. Journal of Physics: Condensed Matter, 2(2):405, 1990.

[33] J. Graf, C. Jozwiak, C. L. Smallwood, H. Eisaki, R. A. Kaindl, D-H. Lee, and A. Lanzara. Nodal quasiparticle meltdown in ultrahigh-resolution pump-probe angle-resolved photoemission. Nat Phys, 7(10):805-809, October 2011.

[34] Christopher L. Smallwood, James P. Hinton, Christopher Jozwiak, Wentao Zhang, Jake D. Koralek, Hiroshi Eisaki, Dung-Hai Lee, Joseph Orenstein, and Alessandra Lanzara. Tracking cooper pairs in a cuprate superconductor by ultrafast angle-resolved photoemission. Science, 336(6085): 1137-1139, 2012.

[35] Bo-Xiao Zheng and Garnet Kin-Lic Chan. Groundstate phase diagram of the square lattice hubbard model from density matrix embedding theory. Phys. Rev. B, 93:035126, Jan 2016.

[36] H. Hafermann, M. I. Katsnelson, and A. I. Lichtenstein. Metal-insulator transition by suppression of spin fluctuations. EPL (Europhysics Letters), 85 (3):37006, 2009.

[37] Junya Otsuki, Hartmut Hafermann, and Alexander I. Lichtenstein. Superconductivity, antiferromagnetism, and phase separation in the twodimensional hubbard model: A dual-fermion approach. Phys. Rev. B, 90:235132, Dec 2014.

[38] Emanuel Gull, Andrew J. Millis, Alexander I. Lichtenstein, Alexey N. Rubtsov, Matthias Troyer, and Philipp Werner. Continuous-time monte carlo methods for quantum impurity models. Rev. Mod. Phys., 83:349-404, May 2011.

[39] Priyanka Seth, Igor Krivenko, Michel Ferrero, and Olivier Parcollet. Triqs/cthyb: A continuoustime quantum monte carlo hybridisation expansion solver for quantum impurity problems. Computer Physics Communications, 200:274 - 284, 2016.

[40] B. Dalla Piazza, M. Mourigal, M. Guarise, H. Berger, T. Schmitt, K. J. Zhou, M. Grioni, and H. M. Rønnow. Unified one-band hubbard model for magnetic and electronic spectra of the parent compounds of cuprate superconductors. Phys. Rev. $B, 85: 100508$, Mar 2012.

[41] Mark Jarrell and J.E. Gubernatis. Bayesian inference and the analytic continuation of imaginarytime quantum monte carlo data. Physics Reports, 269(3):133 - 195, 1996.

[42] Makoto Hashimoto, Inna M. Vishik, Rui-Hua He, Thomas P. Devereaux, and Zhi-Xun Shen. Energy gaps in high-transition-temperature cuprate superconductors. Nat Phys, 10(7):483-495, July 2014.

[43] Ralf Bulla, Theo A. Costi, and Thomas Pruschke. Numerical renormalization group method for quantum impurity systems. Rev. Mod. Phys., 80:395450, Apr 2008.

[44] V Yu Irkhin and MI Katsnelson. Theory of intermediate-valence semiconductors. Sov. Phys. JETP, 63:631, 1986.

[45] Shiro Sakai, Marcello Civelli, and Masatoshi Imada. Hidden fermionic excitation boosting hightemperature superconductivity in cuprates. Phys. Rev. Lett., 116:057003, Feb 2016.

[46] F. Verstraete, V. Murg, and J.I. Cirac. Matrix product states, projected entangled pair states, and variational renormalization group methods for quantum spin systems. Advances in Physics, 57(2): 143-224, 2008.

[47] V. Yu. Irkhin, A. A. Katanin, and M. I. Katsnelson. Robustness of the van hove scenario for high- $T_{c}$ superconductors. Phys. Rev. Lett., 89:076401, Jul 2002.

[48] K. I. Kugel, A. L. Rakhmanov, A. O. Sboychakov, Nicola Poccia, and Antonio Bianconi. Model for phase separation controlled by doping and the internal chemical pressure in different cuprate superconductors. Phys. Rev. B, 78:165124, Oct 2008. 